Tuberc Respir Dis 2013;74:51-55

Copyright(C)2013. The Korean Academy of Tuberculosis and Respiratory Diseases. All rights reserved.

\title{
Acute Eosinophilic Pneumonia
}

Jang Won Sohn, M.D.

Division of Pulmonary Medicine, Department of Internal Medicine, Hanyang University College of Medicine, Seoul, Korea

Acute eosinophilic pneumonia is a severe and rapidly progressive lung disease that can cause fatal respiratory failure. Since this disease exhibits totally different clinical features to other eosinophilic lung diseases (ELD), it is not difficult to distinguish it among other ELDs. However, this can be similar to other diseases causing acute respiratory distress syndrome or severe community-acquired pneumonia, so the diagnosis can be delayed. The cause of this disease in the majority of patients is unknown, even though some cases may be caused by smoke, other patients inhaled dust or drugs. The diagnosis is established by bronchoalveolar lavage. Treatment with corticosteroids shows a rapid and dramatic positive response without recurrence.

Key Words: Pulmonary Eosinophilia; Respiratory Distress Syndrome, Adult; Eosinophils; Pneumonia

\section{Introduction}

The eosinophilic lung diseases (ELD) are a varying group of conditions that are accompanied by increased eosinophils in the lung. In normal condition, eosinophils are found in the lung in very few numbers (less than $2 \%$ on bronchoalveolar lavage [BAL]). ELD show increased lung eosinophils defined by increased number of eosinophils on lung biopsy or greater than $5 \%$ of eosinophils on BAL. Especially in eosinophilic pneumonias, very high ratio of eosinophils in BAL $(>25 \%)$ is presented. The idiopathic eosinophilic pneumonias include simple eosinophilic pneumonia (Loeffler's syndrome), chronic eosinophilic pneumonia (CEP), and acute eosinophilic pneumonia (AEP). Idiopathic AEP was first recognized as a distinct clinical entity in $1989^{1,2}$. It is characterized by large numbers of eosino-

Address for correspondence: Jang Won Sohn, M.D.

Division of Pulmonary Medicine, Department of Internal

Medicine, Hanyang University College of Medicine, 222

Wangsimni-ro, Seongdong-gu, Seoul 133-791, Korea

Phone: 82-2-2220-8351, Fax: 82-2-2298-9183

E-mail: jwsohn@hanyang.ac.kr

Received: Dec. 20, 2012

Revised: Dec. 26, 2012

Accepted: Jan. 4, 2013

(a) It is identical to the Creative Commons Attribution Non-Commercial License (http://creativecommons. org/licenses/by-nc/3.0/). phils infiltration in the interstitium and air space, with rapidly progress to acute respiratory failure.

\section{Characteristics of Eosinophil}

Eosinophils are predominantly a tissue-resident cell and specific leukocyte implicated in immune function for inflammatory reactions to allergic diseases or various infections, especially parasitic helminthes ${ }^{3}$. Eosinophils are under the control of T-lymphocytes. The eosinophil matures in the bone marrow under the action of lymphocyte (Th2) cytokines, especially interleukin (IL)-5, IL-3, and granulocyte-macrophage colony-stimulating factor $^{4,5}$. Tissue-generated cytokines (IL-4, IL-13, and the eotaxins) and adhesion molecules (VCAM-1 and P-selectin) are important in attraction of eosinophils to tissues $^{6}$. The peripheral blood levels of eosinophils depend on balance between production in bone marrow and tissue migration ${ }^{7}$. The eosinophil has eosinophilspecific proteins including major basic protein (MBP), eosinophil cationic protein, eosinophil-derived neurotoxin (or eosinophil-protein X), and the enzymatic protein eosinophil peroxidase and MBP homologue within its granules that facilitates host defense against pathogens such as parasites and fungi. Activation of the eosinophil results in degranulation with the extracellular re- 
lease of the toxic chemical substance. Release of toxic granule proteins and lipid mediators by eosinophils may contribute to tissue damage, dysfunction and promotion of a proinflammatory response resulting in the development of the clinical abnormalities ${ }^{8}$.

\section{Causes and Pathogenetic Factors}

The cause of AEP is most commonly unknown. However, other conditions can cause in an AEP-like clinical symptoms. Many probable stimuli can activate T-lymphocytes to initiate eosinophil accumulation in the lung. Some patients had unusual outdoor activities before onset of disease, such as cave exploration, plant repotting, wood pile moving, smokehouse cleaning, or motocross racing in dusty conditions ${ }^{9-11}$. However, many of these exposures are in the form of single case reports, and so definite causality cannot be fully defined. A causative role of cigarette smoke is well presented. AEP has developed soon after the initiation of smoking in numerous patients especially when starting with large quantities, and re-challenge with cigarette smoking was resulted in recurrence some of them, but tolerance may develop $^{12,13}$. It is likely that inhalation of tobacco smoke or of any nonspecific injurious agent may initiate or contribute to the development of idiopathic acute eosinophilic pneumonia (IAEP) in susceptible individuals. Drugs also have been reported to be as causative factors of AEP. Bacillus Calmette-Guérin vaccination, minocycline, daptomycin, fludarabine, intramuscular progesterone, and sertraline are presented to be associated with AEP. Infections can also present as AEP, including Aspergillus and coccidioidomycosis ${ }^{9}$. Increased levels of a major component of the cell wall of most fungi and also one of the components of cigarette smoke, beta-D-glucan have been reported in BAL fluid of patients with IAEP $^{14}$. So, AEP can be considered as a syndrome that can be a result of any inciting conditions or can be idiopathic. An inhalation exposure history including recent smoking and illegal drugs are necessary.

\section{Clinical Preservation}

IAEP was first reported as a specific clinical entity in 1989. All age groups can be affected with an average of 30 years $^{15,16}$. Men and women are affected equally. In contrast with idiopathic CEP, there is no prior asthma history. Because cases reported throughout the United States, Europe, and Asia, there does not seem to be a regional predilection ${ }^{16}$. Patients with AEP present with rapid onset of cough, tachypnea, and dyspnea in previously healthy individuals of usually within 1 to 5 days of symptom onset. It may also develop subacutely over a few weeks, 12 with no clinical difference between patients seen in a time interval of less than 7 days or 7 to 31 days from the onset. Therefore, even this subacute duration of symptoms is less common, it should not exclude the diagnosis. Patients can progress from mild dyspnea to life-threatening respiratory failure in only a few hours. All patients have nonproductive cough, tachypnea, and dyspnea. Fever is usually present over $38^{\circ} \mathrm{C}$. Pleuritic chest pain is present in frequently, and myalgias are present in about half of patients. At physical examination, tachypnea, tachycardia, and crackles are present in $80 \%$ of patients, and wheezes on auscultation with less often ${ }^{1,2,15,16}$. In 20\% of patients the lungs are clear to auscultation. In a study of AEP in US military personnel, an incidence of AEP was 9.1 per 100,000 person-years $^{17}$. In this study, all patients were smokers.

\section{Bronchoalveolar Lavage}

Early BAL in patients with unexplained diffuse pulmonary infiltrates and respiratory failure is necessary for prompt diagnosis, because AEP patients clinically resemble pneumonia or acute respiratory distress syndrome (ARDS), and with the usual lack of initial blood eosinophilia. AEP showing surprisingly increased eosinophils in BAL fluid of an average percentage of $37 \%$ to $54 \%$ eosinophils at differential cell count, with sterile bacterial cultures ${ }^{11,16}$. We consider that the finding of eosinophil greater than 25\% in BAL can substitute lung biopsy, at least in normal immune patients. In CEP, 
there are increased eosinophils, with lymphocytes and neutrophils present in relatively normal percentages in BAL. Thus, the presence of increased lymphocytes and neutrophils in BAL can be an important finding to help distinguish acute versus CEP in difficult to differentiate cases.

\section{Laboratory Findings}

In initial complete blood count, white blood cell count at presentation usually shows increased leukocytosis with a predominance of neutrophils, with eosinophils only rarely higher than $300 / \mathrm{mm}^{3}$. However, when the patients are followed over the course of hospitalization, the eosinophil count may rise mild to moderate values during the course of disease in most of them. Eosinophilia is also present at pleural fluid and in the sputum. The IgE level can be normal or elevated to levels as high as $2,310 \mathrm{kU} / \mathrm{L}^{18}$. Hypoxemia is present in all patients with severe and refractory to breathing 100\% oxygen in some patients ${ }^{1,16}$. A majority of patients fit the definition of acute lung injury (ALI) or ARDS. Mechanical ventilation is necessary in a majority of patients. In contrast with ALI or ARDS, other organ failure or shock does not occur. Pulmonary function tests are generally not necessary for diagnosis. It can show small airway obstruction and restriction on spirometry. Diffusing capacity is $\operatorname{low}^{18}$. Lung function tests performed after recovery are normal in most patients.

\section{Radiographic Findings}

Chest X-ray shows a pattern consistent with pulmonary edema, with extensive airspace opacity, interlobular septal thickening, and pleural effusions. The infiltrates are diffuse and not peripherally based, unlike radiographs of patients with CEP. Bilateral pleural effusion and Kerley-B lines are common ${ }^{19}$. The chest X-ray returns to normal within 3 weeks, with pleural effusions being the last longer abnormality to disappear. The computed tomography (CT) findings of patients with AEP differed substantially from those of CEP such that, so the two diseases were easily distinguished. CT scans can show diffuse interstitial infiltrates, patchy alveolar infiltrates, or diffuse ground glass infiltrates ${ }^{20}$. Pleural effusion present in at least two thirds of patients is usually bilateral. Bilateral pleural effusion and interlobular septal thickening are highly characteristic of IAEP in a patient with eosinophilic pneumonia.

\section{Pathology}

Lung biopsy is seldom necessary. The main purpose of lung biopsy is to exclude other diseases that can mimic AEP in the proper clinical setting such as fungal infection in immune compromised patients ${ }^{21}$. When done, it shows marked infiltration of eosinophils in the interstitium and in the alveolar spaces together with acute and organizing diffuse alveolar damage ${ }^{16,22}$.

\section{Diagnosis and Differential Diagnosis}

Because clinical presentations of AEP resemble severe community-acquired pneumonia or ARDS, early diagnosis is necessary for prompt treatment. Proper clinical setting with the typical finding of BAL can provide a confident diagnosis of $\mathrm{AEP}^{1,23}$. According to the clinical presentation of AEP, current diagnostic criteria are: 1) acute onset of febrile respiratory manifestations $(\leq 1$ month duration before consultation); 2) bilateral diffuse opacities on chest radiography; 3) hypoxemia, with $\mathrm{PaO}_{2}$ on room air $<60 \mathrm{~mm} \mathrm{Hg}$, and/or $\mathrm{PaO}_{2} / \mathrm{FiO}_{2} \leq 300$ $\mathrm{mm} \mathrm{Hg}$, and/or oxygen saturation on room air $<90 \%$; 4) lung eosinophilia, with $>25 \%$ eosinophils on BAL differential cell count (or eosinophilic pneumonia at lung biopsy); 5) absence of infection, or of other known causes of eosinophilic lung disease (especially exposure to a drug susceptible to induce pulmonary eosinophilia).

The clinical features of this disease are totally different to other ELD. Therefore, it is not difficult to distinguish to other ELD. However, this can be similar other diseases causing ARDS or severe community-acquired pneumonia. Futhermore, the differential diagnosis in- 
cludes drug reaction and fungal infection. In patients with a pertinent exposure history of fungi (Aspergillus and coccidioidomycosis) or parasites (especially strongyloides), cultures, microscopic examination, or serologic studies for them should be undertaken. Newly prescribed or illegal drugs medication history should be taken and suspect drugs removed. An especially important consideration in the differential diagnosis is aspergillus infection, which can mimic AEP but which requires antifungal agents instead of corticosteroids ${ }^{24}$. Because that could be made worse with steroids, a lung biopsy may be required to fully exclude infections in patients with suspected exposure to composted organic material.

\section{Treatment and Prognosis}

Patients with AEP have a rapid and very good response to corticosteroids. Recovery is fast with no significant clinical or imaging abnormalities and without relapse after stopping corticosteroid treatment. Most patients have significant clinical improvement within 2448 hours. Although recovery without corticosteroid treatment may occur, it is not possible to predict spontaneous resolution at the time of initial presentation. So, corticosteroids should be started at the time of diagnosis to all patients without an improving clinical course.

Although the optimal dose and duration of corticosteroids have not been determined, commonly used doses of intravenous methylprednisolone range from 60 to $125 \mathrm{mg}$ every 6 hours are given until respiratory failure resolves ${ }^{25}$. In recovering course, the patient can be changed to oral prednisone, and can be tapered off over several weeks ${ }^{16,22}$. Unlike patients with CEP, recurrence of AEP is exceedingly rare after completing treatment of corticosteroids. Patients should ideally be managed in an intensive care unit in the early course of disease. Approximately two-thirds of patients require mechanical ventilation. Although IAEP often presents clinically like ALI or ARDS, its prognosis is far better. However, death from respiratory failure can occur if the disease is not early diagnosed and treated promptly.

\section{References}

1. Allen JN, Pacht ER, Gadek JE, Davis WB. Acute eosinophilic pneumonia as a reversible cause of noninfectious respiratory failure. N Engl J Med 1989;321:569-74.

2. Badesch DB, King TE Jr, Schwarz MI. Acute eosinophilic pneumonia: a hypersensitivity phenomenon? Am Rev Respir Dis 1989;139:249-52.

3. Blanchard C, Rothenberg ME. Biology of the eosinophil. Adv Immunol 2009;101:81-121.

4. Allen JN, Liao Z, Wewers MD, Altenberger EA, Moore SA, Allen ED. Detection of IL-5 and IL-1 receptor antagonist in bronchoalveolar lavage fluid in acute eosinophilic pneumonia. J Allergy Clin Immunol 1996;97: 1366-74.

5. Katoh S, Matsumoto N, Matsumoto K, Fukushima K, Matsukura S. Elevated interleukin-18 levels in bronchoalveolar lavage fluid of patients with eosinophilic pneumonia. Allergy 2004;59:850-6.

6. Ponath PD, Qin S, Post TW, Wang J, Wu L, Gerard $\mathrm{NP}$, et al. Molecular cloning and characterization of a human eotaxin receptor expressed selectively on eosinophils. J Exp Med 1996;183:2437-48.

7. Wenzel SE, Schwartz LB, Langmack EL, Halliday JL, Trudeau JB, Gibbs RL, et al. Evidence that severe asthma can be divided pathologically into two inflammatory subtypes with distinct physiologic and clinical characteristics. Am J Respir Crit Care Med 1999;160:1001-8.

8. Daimon T, Tajima S, Oshikawa K, Bando M, Ohno S, Sugiyama Y. KL-6 and surfactant proteins A and D in serum and bronchoalveolar lavage fluid in patients with acute eosinophilic pneumonia. Intern Med 2005; 44:811-7.

9. Allen J. Acute eosinophilic pneumonia. Semin Respir Crit Care Med 2006;27:142-7.

10. Rom WN, Weiden M, Garcia R, Yie TA, Vathesatogkit $\mathrm{P}$, Tse DB, et al. Acute eosinophilic pneumonia in a New York City firefighter exposed to World Trade Center dust. Am J Respir Crit Care Med 2002;166:797800.

11. Philit F, Etienne-Mastroianni B, Parrot A, Guerin C, Robert D, Cordier JF. Idiopathic acute eosinophilic pneumonia: a study of 22 patients. Am J Respir Crit Care Med 2002;166:1235-9.

12. Shintani H, Fujimura M, Ishiura Y, Noto M. A case of cigarette smoking-induced acute eosinophilic pneumonia showing tolerance. Chest 2000;117:277-9.

13. Uchiyama H, Suda T, Nakamura Y, Shirai M, Gemma $\mathrm{H}$, Shirai $\mathrm{T}$, et al. Alterations in smoking habits are as- 
sociated with acute eosinophilic pneumonia. Chest 2008;133:1174-80.

14. Kawayama T, Fujiki R, Honda J, Rikimaru T, Aizawa $\mathrm{H}$. High concentration of (1->3)-beta-D-glucan in BAL fluid in patients with acute eosinophilic pneumonia. Chest 2003;123:1302-7.

15. Hayakawa H, Sato A, Toyoshima M, Imokawa S, Taniguchi M. A clinical study of idiopathic eosinophilic pneumonia. Chest 1994;105:1462-6.

16. Pope-Harman AL, Davis WB, Allen ED, Christoforidis AJ, Allen JN. Acute eosinophilic pneumonia: a summary of 15 cases and review of the literature. Medicine (Baltimore) 1996;75:334-42.

17. Shorr AF, Scoville SL, Cersovsky SB, Shanks GD, Ockenhouse CF, Smoak BL, et al. Acute eosinophilic pneumonia among US Military personnel deployed in or near Iraq. JAMA 2004;292:2997-3005.

18. Ogawa H, Fujimura $M$, Matsuda $T$, Nakamura $H$, Kumabashiri I, Kitagawa S. Transient wheeze: eosinophilic bronchobronchiolitis in acute eosinophilic pneumonia. Chest 1993;104:493-6.

19. King MA, Pope-Harman AL, Allen JN, Christoforidis GA, Christoforidis AJ. Acute eosinophilic pneumonia: radiologic and clinical features. Radiology 1997;203: 715-9.

20. Johkoh T, Muller NL, Akira M, Ichikado K, Suga M, Ando M, et al. Eosinophilic lung diseases: diagnostic accuracy of thin-section CT in 111 patients. Radiology 2000;216:773-80.

21. Ricker DH, Taylor SR, Gartner JC Jr, Kurland G. Fatal pulmonary aspergillosis presenting as acute eosinophilic pneumonia in a previously healthy child. Chest 1991;100:875-7.

22. Tazelaar HD, Linz LJ, Colby TV, Myers JL, Limper AH. Acute eosinophilic pneumonia: histopathologic findings in nine patients. Am J Respir Crit Care Med 1997;155: 296-302.

23. Cottin V, Cordier JF. Eosinophilic pneumonias. Allergy 2005;60:841-57.

24. Trawick D, Kotch A, Matthay R, Homer RJ. Eosinophilic pneumonia as a presentation of occult chronic granulomatous disease. Eur Respir J 1997;10:2166-70.

25. Rhee CK, Min KH, Yim NY, Lee JE, Lee NR, Chung MP, et al. Clinical characteristics and corticosteroid treatment of acute eosinophilic pneumonia. Eur Respir J 2013;41:402-9. 